\title{
Weak Gravity Conjecture for Noncommutative Field Theory
}

\author{
Qing-Guo Huang ${ }^{1}$ and Jian-Huang She ${ }^{2}$ \\ 1 School of physics, Korea Institute for Advanced Study, \\ 207-43, Cheongryangri-Dong, Dongdaemun-Gu, Seoul 130-722, Korea \\ 2 Institute of Theoretical Physics, Academia Sinica \\ P. O. Box 2735, Beijing 100080, P. R. China \\ huangqg@kias.re.kr \\ jhshe@itp.ac.cn
}

\begin{abstract}
We investigate the weak gravity bounds on the $\mathrm{U}(1)$ gauge theory and scalar field theories in various dimensional noncommutative space. Many results are obtained, such as the upper bound on the noncommutative scale $g_{Y M} M_{p}$ for four dimensional noncommutative $\mathrm{U}(1)$ gauge theory. We also discuss the weak gravity bounds on their commutative counterparts. For example, our result on 4 dimensional noncommutative U(1) gauge theory reduces in certain limit to its commutative counterpart suggested by Arkani-Hamed et.al at least at tree-level.
\end{abstract}

August, 2006 
By now, low energy effective field theory has become the cornerstone in our description of nature. One of the embarrassing features of effective field theory is that couplings of the fields are determined phenomenologically, beyond a first principal derivation. It is generally expected that a quantum theory of gravity would shed some light on this issue. Recently in searching for criteria to distinguish string landscape, which has a well-defined UV theory, from the swampland, which cannot be completed to a fully self-consistent theory, it was proposed in [1,2] that gravity induces some constraints on the quantum field theory.

Field theory is generally well defined in the weak coupling limit. However the situation changes when we take gravity into account, since in the weak coupling limit the nonperturbative objects in the field theory are very heavy and the gravitational effects on them are significant. In [2], requiring that the magnetic monopole should not collapse to be a black hole yields a new nontrivial UV cutoff for the U(1) gauge theory. Some related works are discussed in [3/10].

In this note we generalize the results of [2] to noncommutative gauge theory and scalar field theory. In particular, the scalar field theory in three or higher dimensions, which has no solitonic solutions in commutative space, will possess such solutions when promoted to noncommutative space. Thus the mechanism proposed in [2] can be more generically used for noncommutative field theories. The investigation of noncommutative field theory can also give us some hints on conventional field theory.

First we consider in this note type IIB string theory with a D3-brane placed in a nonzero B field with components along the space-space directions. It is convenient to define the open string metric $G_{i j}$, constant asymmetric matrix $\theta^{i j}$, gauge coupling $g_{Y M}$ through closed string metric $g_{i j}$, B-field $B_{i j}$ and string coupling $g_{s}$ as follows:

$$
\begin{aligned}
G_{i j} & =g_{i j}-\left(2 \pi \alpha^{\prime}\right)^{2}\left(B g^{-1} B\right)_{i j} \\
\theta^{i j} & =-\left(2 \pi \alpha^{\prime}\right)^{2}\left(\frac{1}{g+2 \pi \alpha^{\prime} B} B \frac{1}{g-2 \pi \alpha^{\prime} B}\right)^{i j} \\
g_{Y M}^{2} & =2 \pi g_{s}\left(\operatorname{det}\left(1+2 \pi \alpha^{\prime} g^{-1} B\right)\right)^{\frac{1}{2}} .
\end{aligned}
$$

We choose the constant B-field to be $\frac{1}{2} B d x^{1} \wedge d x^{2}$, with the D3-brane lying in 0123 directions and set the open string metric to be Euclidean $G_{i j}=\delta_{i j}$, thus we have

$$
B=\frac{\theta}{\left(2 \pi \alpha^{\prime}\right)^{2}+\theta^{2}}
$$


and

$$
g_{s}=g_{Y M}^{2} \frac{\alpha^{\prime}}{\sqrt{\left(2 \pi \alpha^{\prime}\right)^{2}+\theta^{2}}} .
$$

According to [1], in the limit $\alpha^{\prime} \rightarrow 0$ with $G, \theta, g_{Y M}^{2}$ fixed, closed string degrees of freedom as well as massive open string degrees of freedom decouple, and the brane reduces to a field theory, namely $\mathcal{N}=4$ super Yang-Mills theory on a noncommutative space with

$$
\left[x^{i}, x^{j}\right]=i \theta^{i j} .
$$

Here we need to stress that we only pay attention to the constraints on the field theory in the noncommutative space and don't need to take care of the origin of the noncommutative space.

It is also tempting to go in the reversed direction, that is, to start with the decoupled field theory, and turn on gravitational effects gradually, and ask about the constraints imposed by gravity upon the parameters in the field theory [2]. In [2] it was shown that for a $\mathrm{U}(1)$ gauge theory in commutative space, unless the cutoff satisfies

$$
\Lambda \leq g_{Y M} M_{4}
$$

where $M_{4} \sim G_{4}{ }^{-1 / 2}$ is the Planck scale in four dimensions, the magnetic monopole would collapse to a black hole, ruining the validity of the effective field theory. The advantage of the methods used in [2] is that the constraint does not depend on the particular form of the high energy completion of the field theory, of which one still lacks of full control. Subsequent work in this direction includes [1, 6].

The U(1) NCYM also has a monopole solution, but with a semi-infinite string attached to it [12]. In fact, the solution is everywhere non-singular and the energy density localizes along a half-line. The tension of the string is given by

$$
T=\frac{2 \pi}{g_{Y M}^{2} \theta}
$$

This soliton is qualitatively different from their commutative counterpart, which is pointlike, with no strings detached.

When one turns on gravity, a natural question to ask is how do noncommutative field theories couple to gravity, or should they couple to conventional gravity or some noncommutative version. In our paper, we only take care of the IR behavior of gravity 
and thus we assume the noncommutative field theories are coupled to the conventional gravity.

Another question is whether the field theory solitons persist their existence when quantum gravity effects are turned on. For general cases we still do not have a general proof of their existence. But for the case at hand, which has a natural embedding in string theory, this question has a simple positive answer. The soliton solution, a monopole with a string detached, is just a D1-string ending on a D3-brane. And its tension can also be calculated from the brane configuration to be exactly (6) in [12].

From the point of view of an asymptotic observer, the effect of the string is to produce a deficit angle $8 \pi G_{4} T$ in spacetime. Requiring that the deficit angle is less than $2 \pi$ yields

$$
8 \pi G_{4} T \leq 2 \pi
$$

Ignoring numerical factors, one gets

$$
g_{Y M}^{2} \theta \geq G_{4} \sim M_{4}^{-2}
$$

When space-space becomes noncommutative the weak gravity conjecture yields a lower bound on the gauge coupling; or equivalently, upper bound on the noncommutative scale $M_{n c}=\theta^{-1 / 2}$ with

$$
M_{n c} \leq g_{Y M} M_{4}
$$

Naively to go to the commutative theory, one chooses the cutoff scale $\Lambda$ to be lower than the noncommutative scale $\Lambda \leq M_{n c}$, which leads to (5). However, this is reliable only in the tree-level approximation, because of the non-analytic behavior of $\theta$ 13 in noncommutative gauge theory. In supersymmetric gauge theories the logarithmic divergences at small values of noncommutative momenta typically appear. One can only expect that in $\mathcal{N}=4$ supersymmetric gauge theory even the logarithmic divergences do not occur, and thus this noncommutative supersymmetric gauge theory reduces to its commutative counterpart when $\Lambda<M_{n c}$.

It is interesting to ask about the limiting case with zero or small B field. We start from the field theory limit, where $\alpha^{\prime} \rightarrow 0, \theta$ finite, $B \sim \frac{1}{\theta}$, thus small values of $\mathrm{B}$ correspond to large noncommutative parameter $\theta$. When gravitational effects are still weak and $\alpha^{\prime}$ small (compared to $\theta$ ), small values of $\mathrm{B}$ correspond to large values of $\theta$. Our constraint for the validity of the noncommutative field theory doesn't contradict the existence of vacua with $B=0$ or very small value of $B$. 
We can also consider gauge theory in $2+1$ dimensions. It is interesting at with noncommutativity, even pure $U(1)$ gauge theory admits finite energy solitonic solutions [14]. The energy is

$$
E=\frac{2 \pi}{g_{3}^{2}} \int d t \operatorname{Tr} \frac{1}{2} F^{2},
$$

where $g_{3}$ is the $\mathrm{U}(1)$ gauge coupling in three dimensions. With vortex number $n=\operatorname{Tr} F^{2}$, the simplest nonsingular fluxon solutions have energy

$$
M_{f}=\frac{\pi n}{g_{3}^{2} \theta}
$$

When gravity is turned on, we similarly get a constraint for the gauge coupling

$$
\frac{g_{3}^{2} \theta}{n} \geq G_{3}
$$

here $G_{3}$ is the Newton coupling constant in three dimensions. In the $n=1$ sector, $M_{n c}=$ $\theta^{-1 / 2} \leq g_{3} / \sqrt{G_{3}}$. Similarly we conjecture that $\Lambda \leq g_{3} / \sqrt{G_{3}}$ for the commutative gauge theory. Instead of the dimensional gauge coupling $g_{3}$ we use the dimensionless gauge coupling $\tilde{g}_{3}=\Lambda^{-1 / 2} g_{3}$ 国 and thus

$$
\Lambda \leq \tilde{g}_{3}^{2} / G_{3} \sim \tilde{g}_{3}^{2} M_{3}
$$

Naturalness says that the dimensionless coupling is roughly $\mathcal{O}(1)$ and thus $\Lambda \leq M_{3}$. If there is a UV theory including gravity beyond the this effective $\mathrm{U}(1)$ theory, the value of $\tilde{g}_{3}$ is decided by this UV theory and the matching condition should satisfies $\Lambda \sim \tilde{g}_{3}^{2} M_{3}$. A similar argument is also reliable for the result in [2].

Next, we consider the noncommutative scalar field theory with polynomial potential in $\phi$. According to Derrick's theorem [15], there is no finite energy solitonic solutions for commutative scalar field theories in three and more dimensions. But noncommutativity provides a natural mechanism for stabilizing objects of size $\sqrt{\theta}$. For sufficiently large $\theta$, there are solitonic solutions [14,16,17] in odd dimensional scalar noncommutative field theories with nice potentials. In the following, we use the notation of [14] and show some concrete examples.

1 In [10], the dimensionless gauge coupling is defined as $\tilde{g}_{3}^{\prime}=M_{3}^{-1 / 2} g_{3}$ and thus $\Lambda \leq \tilde{g}_{3}^{\prime} M_{3}$. Since $M_{3}$ is not a reasonable scale if $\tilde{g}_{3}<1$, we don't use the definition in [10]. 
We consider a scalar field theory with Euclidean action in $2+1$ dimensions

$$
S=\int d^{3} x \sqrt{g}\left(\frac{1}{2} g^{i j} \partial_{i} \phi \partial_{j} \phi+V(\phi)\right) .
$$

In the canonically commuting noncommutative coordinates

$$
\begin{aligned}
& z=\frac{x^{1}+i x^{2}}{\sqrt{\theta}} \\
& \bar{z}=\frac{x^{1}-i x^{2}}{\sqrt{\theta}},
\end{aligned}
$$

the energy is

$$
E=\int d^{2} z\left(\frac{1}{2}(\partial \phi)^{2}+\theta V(\phi)\right)
$$

When $\theta V$ is large, the potential energy dominates and we can find an approximate solitonic solution by solving the equation $\frac{d V}{d \phi}=0$.

For an illustrating example, consider a cubic potential

$$
V(\phi)=\frac{1}{2} m^{2} \phi^{2}+\frac{1}{3} \lambda_{3} \phi^{3} .
$$

Solving $\frac{d V}{d \phi}=0$ yields $\phi=0,-m^{2} / \lambda_{3}$. With large enough $\theta\left(\theta>1 / m^{2}\right)$, the simplest solitonic solution has energy [14,17

$$
E_{0}=2 \pi \theta V\left(-m^{2} / \lambda_{3}\right)=\frac{\pi m^{6} \theta}{3 \lambda_{3}^{2}} .
$$

We can similarly turn on gravity, and this soliton will create a deficit angle in spacetime, which should not exceed $2 \pi$

$$
8 \pi G_{3} E_{0} \leq 2 \pi .
$$

Neglecting order 1 coefficients in the subsequent analysis, we get

$$
\frac{\lambda_{3}{ }^{2}}{m^{6}} \geq G_{3} \theta .
$$

The cubic coupling should also be nonzero when space-space is sufficiently noncommutative. Note that $\lambda_{3}$ has dimension of length square and its lower bound is determined by both gravitational effect and noncommutative effect. In the absence of gravity $\left(G_{3} \rightarrow 0\right)$, the bound is trivial. Taking into account the condition for the existence of the noncommutative soliton $\theta>1 / m^{2}$ yields

$$
\frac{1}{\theta} \leq m^{2} \leq \frac{\lambda_{3}}{\sqrt{G_{3}}}
$$


in the noncommutative field theory. The right part of eq. (21) is independent on the noncommutative parameter. The dimensional quantities depend on the scale $\Lambda$. Define the dimensionless variables $\tilde{m}=\Lambda^{-1} m$ and $\tilde{\lambda}_{3}=\Lambda^{-3 / 2} \lambda_{3}$. Thus eq. (21) becomes

$$
\Lambda \leq\left(\frac{\tilde{\lambda}_{3}}{\tilde{m}^{2}}\right)^{2} M_{3}
$$

For $\Lambda \geq m, \tilde{m} \leq 1$; otherwise the lightest quanta cannot be excited. Naturalness implies $\tilde{\lambda}_{3} \sim \mathcal{O}(1)$ and eq. (22) becomes $\Lambda \leq M_{3}$, which can be accepted by any effective field theorist. The bound on the scale in eq. (22) becomes significant only for the weak coupled situation.

The authors in [8] cited our results to support their conjectures. In fact, this mechanism is very generic. One can consider for example another potential of the form

$$
V=-\frac{\lambda_{6}}{6} \phi^{6}+\frac{\lambda_{8}}{8} \phi^{8}+\frac{1}{24} \frac{\lambda_{6}^{4}}{\lambda_{8}^{3}}
$$

where we add the last term in order that the potential at the global minimum equals zero. For the potential (23), the global minima are located at $\phi= \pm \sqrt{\lambda_{6} / \lambda_{8}}$ and the effective mass for the field theory about one of the minima is $m_{\text {eff }}=\sqrt{2 \lambda_{6}^{3} / \lambda_{8}^{2}}$. When $\theta m_{\text {eff }}^{2}>1$, there is a noncommutative soliton with energy

$$
E \sim \frac{\theta \lambda_{6}^{4}}{\lambda_{8}^{3}}
$$

and subsequently the constraint reads

$$
\frac{\lambda_{8}^{3}}{\lambda_{6}^{4}} \geq G \theta
$$

Combining the condition for the existence of the noncommutative soliton yields

$$
\frac{1}{\theta} \leq \frac{\lambda_{6}^{3}}{\lambda_{8}^{2}} \leq \frac{\lambda_{6}^{2}}{\lambda_{8}} M_{3}
$$

The first term in the potential (23) is marginal. Define the dimensionless variable $\tilde{\lambda}_{8}=\Lambda \lambda_{8}$. The right part of eq. (26) becomes

$$
\frac{\lambda_{6}}{\lambda_{8}} \leq M_{3}, \quad \text { or, } \quad \Lambda \leq \frac{\tilde{\lambda}_{8}}{\lambda_{6}} M_{3}
$$


For a general potential, the above calculations can be easily generalized to give a constraint on the couplings involved if there is a solitonic solution.

Because of the absence of a principle to constraint the shape of the potential for the scalar field, we investigate the weak gravity constraint on the scalar field theory case by case. Here we also need to remind the reader that the noncommutative scalar field doesn't simply reduce to commutative scalar field theory for low momentum [18], because of the UV/IR mixture. Whether such effects will destroy the constraints on general scalar field theory certainly needs more study, even though the noncommutative parameter $\theta$ doesn't appear in eq. (22) and (27). On the other hand, the conjecture in 8 seems too strong. For example, there is no solitonic solution for the $2+1$ dimensional scalar field theory with potential $V=\frac{1}{2} m^{2} \phi^{2}+\frac{\lambda_{4}}{4} \phi^{4}$ and with $\lambda_{4}>0$ if $m^{2}>0$, even in noncommutative space. There is no evidence to support the conjecture with $\lambda_{4} / m^{2} \geq G_{N}$ in [8]. But for $m^{2}=-\mu^{2}<0$, the potential is just the potential of Higgs field in the standard model where $\mu / \sqrt{\lambda_{4}}$ is just the electroweak energy scale. Requiring that the electroweak energy scale should be lower than the Planck scale $1 / \sqrt{G_{4}}$ leads to $\lambda_{4} / \mu^{2} \geq G_{4}$, which is a trivial result.

In this short note we proposed some conjectures on the noncommutative gauge theory and scalar field theory and related results for their commutative counterparts. The constraints on the noncommutative scalar field is only in odd dimensional spacetime. However we need to keep in mind that gravity does not contain propagating degrees of freedom in three dimensions. We have no evidence to support these constraints on the scalar field theories in four dimensions. On the other hand, whether the noncommutative solitonic solutions exist when the deficit angle approaches to $2 \pi$ in three dimensions is still an open question. But we believe that the magnetic monopole in $\mathrm{U}(1)$ gauge theory should collapse to be a black hole when the Newton coupling constant in four dimensions is large enough because of the conservation of the energy and the magnetic charge. However there is not a corresponding charge for the scalar field theory. The constraints on the scalar field theories need more study in the future. A more ambitious plan is to investigate the implication on the inflaton potential from the viewpoint of weak gravity conjecture on the scalar field theory.

Acknowledgments.

We would like to thank M. Li, F.L. Lin, W. Song, J.B. Wu and X.H. Wu for useful discussions. 


\section{References}

[1] C. Vafa, hep-th/0509212.

[2] N. Arkani-Hamed, L. Motl, A. Nicolis and C. Vafa, hep-th/0601001.

[3] S. Kachru, J. McGreevy and P. Svrcek, JHEP 0604(2006)023, hep-th/0601111.

[4] M. Li, W. Song, T. Wang, hep-th/0601137.

[5] H. Ooguri and C. Vafa, hep-th/0605264.

[6] Q.G. Huang, M. Li, W. Song, hep-th/0603127; Q.G. Huang, hep-th/0610106.

[7] Y. Kats, L. Motl, M. Padi, hep-th/0606100.

[8] M. Li, W. Song, Y.S. Song and T. Wang, hep-th/0606011; A. Medved, hep-th/0611196.

[9] M.R. Douglas, Z. Lu, hep-th/0509224; B.S. Acharya, M.R. Douglas, hep-th/0606212.

[10] T. Banks, M. Johnson and A. Shomer, hep-th/0606277.

[11] N. Seiberg, E. Witten, JHEP 9909 (1999) 032, hep-th/9908142.

[12] D.J. Gross and N.A. Nekrasov, JHEP 0007(2000)034, hep-th/0005204; D.J. Gross and N.A. Nekrasov, JHEP 0010 (2000) 021, hep-th/0007204.

[13] A. Matusis, L. Susskind and N. Toumbas, JHEP 0012(2000)002, hep-th/0002075.

[14] M.R. Douglas, N.A. Nekrasov, Rev.Mod.Phys. 73(2001)977, hep-th/0106048.

[15] G.M. Derrick, J.Math.Phys. 5(1964)1252.

[16] R. Gopakumar, S. Minwalla and A. Strominger, JHEP 0005(2000)020, hep-th/0003160.

[17] J.A. Harvey, hep-th/0102076.

[18] S. Minwalla, M.V. Raamsdonk and N. Seiberg, JHEP 0002(2000)020, hep-th/9912072. 